\title{
Continued progress of preoperative therapy for rectal cancer
}

The indications for adjuvant therapy for rectal cancer are based on the patterns of failure after surgery. Despite radical surgery, local-regional failure occurs frequently in patients with transmural or node-positive rectal cancers. The incidence of local failure in the pelvis increases from less than $10 \%$ in stages T1-2NOMO to $15 \%$ to $30 \%$ in stages T3NOMO and T1-2N1M0, and is as high as $40 \%$ to $60 \%$ in stages T3-4N1-2M $0^{1}$. Much of the recent debate in adjuvant therapy of rectal cancer has centered on the merits of preoperative versus postoperative adjuvant therapy. In some clinical situations, more than one approach may be acceptable. Their selection depends on factors such as tumor histology, size, location, mobility, anatomic constraints, intercurrent medical disease, and the technical expertise of the surgeon and radiation oncologist.

Since the National Cancer Institute Consensus Conference in $1990^{2}$, postoperative combined modality therapy has been considered, at least in the United States, the standard approach as adjuvant treatment for patients with stages II and III rectal cancer. The primary advantage with this approach is pathologic staging and accurate selection of patients for subsequent adjuvant treatment. The primary disadvantages include an increased toxicity due to larger amount of small bowel in the radiation field and the perineal scar if the patient has undergone an abdominal perineal resection, and a potentially hypoxic postsurgical bed which decreases tumor sensitivity to radiation.

On the other hand, the European experience with adjuvant therapy has largely focused on preoperative radiation. Tumor downstaging can improve not only resectability of the tumor but also expand the potential for sphincter preservation options. There is less likelihood of tumor seeding at the time of surgery, and the absence of pelvic fibrosis can considerably minimize potential damage to small bowel and the pelvic viscera. Several trials in the early 90's reported a significant improvement in local control and survival rates with relatively moderate doses of preoperative radiation therapy ${ }^{3-5}$. There was a rapid, but

Rev Oncología 2001; 3: 281-283 incomplete, move from postoperative to preoperative radiotherapy when the results of the Upsala trial became available ${ }^{6}$ and, in fact, this led to the French Consensus Conference held in 1994 recommending the use of preoperative radiotherapy as the standard therapy for rectal cancer in France?

From the viewpoint of sphincter preservation, the advantage of preoperative therapy is to decrease the volume of the primary tumor. When the tumor is located in close proximity to the dentate line, this decrease in tumor volume may allow the surgeon to perform a sphincter-preserving procedure that would not otherwise be possible. If quality of life outcome is considered in patients with rectal cancer, preservation of the sphincter function is an important endpoint in itself. The disadvantage of preoperative radiation therapy is the potential of overtreating patients with either early-stage (pT1-2N0) or metastatic disease. Nevertheless, with a careful rectal digital examination, and with the aid of improved imaging techniques such as endorectal ultrasound, computed tomography or magnetic resonance imaging, the number of patients who are overtreated is decreased ${ }^{8,9}$.

Controversy still exists about the dose and timing of radiation therapy in the preoperative setting. The publication of the Swedish Rectal Cancer Trial ${ }^{10}$ revealed a significant improvement in local control and survival with intensive short-course preoperative radiation, typically, 25 Gy in 5 fractions, followed by surgery 1 week later. The recent meta-analysis of 14 trials, mainly from European centers, corroborates the benefit in survival with the intensive short-course preoperative radiation ${ }^{11}$. In contrast, conventional doses and techniques of radiation, usually combined with chemotherapy, are recommended in United States. These include multiple-field techniques to a total dose of 45 to $50.4 \mathrm{~Gy}$ at 1.8 or $2 \mathrm{~Gy} /$ fraction, followed by surgery 4 to 8 weeks after the completion of radiation ${ }^{12-14}$. According to these authors, this design allows for the recovery from the acute side effects of radiation and enhances tumor downstaging. However, this intense regimen may cause more postoperative or long-term complications, and it certainly requires more resources and a greater amount of time spent in treatment. 
A study comparing both preoperative regimens would clarify data.

More recently, there have been substantial changes in the surgical therapy of rectal cancer. Total mesorectal excision (TME) has been accepted by surgical oncologists as the standard of care, since it can decrease local recurrence rate to $5 \%{ }^{15}$, but it is not clear whether these excellent results can be achieved in the general oncology population. Some physicians content that adjuvant therapy is not necessary if patients undergo resection with a TME; however, it must be emphasized that TME series include patients with T1-2N0 disease and allow identification and exclusion of patients with more advanced disease, compared with patients treated in the adjuvant trials in which more conventional surgery is performed. The recently published work of the Dutch Colorectal Cancer Group investigating the value of adjuvant radiation therapy when total mesorectal excision was performed by specially trained surgeons, has thrown light in this matter ${ }^{16}$. The preliminary results of this multicenter randomized trial show that radiation therapy, given preoperatively as a short course of 5 Gy on each of 5 days, significantly reduces the risk of local recurrence even when administered to patients who underwent total mesorectal excision. These 2-year data suggest that not all local recurrences are attributable to suboptimal surgical technique; in fact, some of them would be best explained by the biological futures of the tumor. The study leaves open the question of which patients benefit most from adjuvant treatment and what type of treatment should be given. To optimize the therapeutic ratio of all adjuvant treatments, it seems appropriate to focus our attention on defining benefits precisely and on selecting treatment options according to risk. For patients with early-stage (T1-2 N0) disease, surgery could be performed first. Either radical surgery or local excision plus postoperative radiotherapy seem to obtain similar results ${ }^{17}$. Given the morbidity of standard surgery, the use of the conservative approach of local excision plus postoperative adjuvant therapy as the primary therapy for selected cases of rectal cancer is appealing. Only for technical reasons, when the tumor is close to the dentate line, preoperative radiation would be justified in order to allow a conservative surgical treatment. Adjuvant chemotherapy can be reserved for those patients with adverse pathologic findings such as high grade tumor or the presence of vascular or lymphatic space invasion.

For patients with transmural resectable (T3-4, N0-1) disease surgery plus preoperative radiation can be recommended. Improved tolerance and better local control when using preoperative versus postoperative irradiation was observed in the only published randomized trial ${ }^{6}$. Our 10-year experience support the superiority of the preoperative adjuvant approach, in terms of improved local control and survival, using moderate dose of radiation ( $30 \mathrm{~Gy}$ in 10 fractions) ${ }^{18}$. Further selection of patients can be done through the use of prognostic factors. For example, subgroup analyses in studies of preoperative treatment have demonstrated more benefit for patients with tumors in the distal rectum or with fixed tumors ${ }^{19}$. Moderate doses of radiation (40 Gy or equivalent) would be appropriate for mobile tumors while higher doses (50 Gy or equivalent) are probably needed for fixed cancers. The addition of chemotherapy can further decreases local recurrence. On the basis of extrapolation from the postoperative combined-modality therapy trials, preoperative radiochemotherapy with continuous infusion of 5-FU rather than radiation therapy alone is recommended for unfavorable subgroups. However, the question of whether preoperative combined-modality therapy is more effective than preoperative radiation therapy is under investigation in a randomized trial from the European Organization for Research and Treatment of Cancer. In the context of sphincter preservation surgery, preoperative chemoradiation followed by full-thickness local excision is also feasible for selected T3 distal rectal cancers ${ }^{20}$.

Finally, preoperative radiochemotherapy has a wellestablished role in the management of locally advanced primary or locally recurrent carcinomas that are technically unresectable. An increasing number of studies using this approach report resectability rates as high as $90 \%$ and local control rates of $60 \%$ to $80 \% \%^{21-23}$. Doses in excess of 50 Gy are recommended for the advanced disease, preferably with conventional fractionation scheme of $2 \mathrm{~Gy} /$ day. The concomitant administration of continuous infusion of 5-FU or some of the oral prodrugs, such as tegafur, UFT or capecitabina enhance radiation-inducing downstaging and are extensively used. Irinotecan and oxaliplatin have also substantial radiation-sensitizing properties, which we can likely exploit in the preoperative setting. Moreover, biological agents such as proteosome inhibitors and antiangiogenesis agents can also produce remarkable levels of radiation sensitization. An important factor in the advanced disease is to allow a minimal interval of 4 to 6 , and preferably 8 , weeks between preoperative treatment and surgery to obtain maximal tumor reduction ${ }^{24}$.

The challenge for today's clinician is to understand that rectal cancer represents a spectrum of disease stages and the clinical parameters of disease presentation need to be combined with an enlightened patient management strategy to optimize results. To answer some pending questions about preoperative therapy patients with rectal cancer should still be entered in prospective clinical trials. Direct comparisons need to be done between: 1) short-course and long-course preoperative radiation; 2) preoperative radiation alone and radiation combined with chemo- 
therapy, and 3) total mesorectal excision and conventional surgery, looking always at survival and quality of life. The improvement on molecular biology knowledge, in the other hand, may allow us in the future to identify molecular tumor factors for patient prognosis and prediction of response to specific therapies. Meanwhile, clinicians must select a reasonable treatment approach according to the best available evidence.

Laura Cerezo Padellano

Department of Radiation Oncology. Hospital Universitario de La Princesa. Madrid (Spain)

\section{References}

1. Minsky BD, Mies C, Recht A, et al. Resectable adenocarcinoma of the rectosigmoid and rectum: Patterns of failure and survival. Cancer 1988; 61: 1.408-1.416.

2. National Institute of Health Consensus Conference. Adjuvant therapy for patients with colon and rectal cancer: JAMA 1990; 264: 1.444-1.450.

3. Gerard A, Buyse M, Nordlinger B, Loygue J. Preoperative radiotherapy as adjuvant treatment in rectal cancer. Ann Surg 1988; 208: 606-614.

4. James RD, Haboubi N, Schofield PF, Mellor M, Salhab N. Prognostic factors in colorectal carcinoma treated by preoperative radiotherapy and immediate surgery. Dis Colon Rectum 1991; 34: 546-551.

5. Vázquez de la Torre M, Cerezo L, Navascués J M, et al. Radioterapia preoperatoria en el adenocarcinoma de recto: nuestra experiencia. Oncología 1994; 22: 17-19.

6. Pahlman L, Glimelius B. Pre or post-operative radiotherapy in rectal and rectosigmoid carcinoma: Report from a randomized multicenter trial. Ann Surg 1990; 211: 187-195.

7. Conference de Consensus, Paris. Le choix therapeutique dans le cancer du rectum. Le Concours Médical, January 1995; 36-42.

8. Hunerbein M, Schlag PM. Three-dimensional endosonography for staging of rectal cancer. Ann Surg 1997; 25: 432-438.

9. Hadfield MB, Nicholson AA, MacDonald AW, et al. Preoperative staging of rectal carcinoma by magnetic resonance imaging with a pelvic phase-array coil. Br J Surg 1997; 84: 529-531.

10. Swedish Rectal Cancer Trial. Improved survival with preoperative radiotherapy in resectable rectal cancer. N Engl J Med 1997; 336: 980-987.

11. Camma C, Giunta M, Fiorica F, et al. Preoperative radiotherapy for resectable rectal cancer. A meta-analysis. JAMA 2000; 284: 1.008-1.015.
12. Minsky BD, Cohen A, Enker W, et al. Pre-operative 5-FU, low-dose leucovorin, and concurrent radiation therapy for rectal cancer. Cancer 1994; 73: 273-278.

13. Grann A, Feng C, Wong D, et al. Preoperative combined modality therapy for clinically resectable T3 rectal adenocarcinoma. Int J Radiat Oncol Biol Phys 2001; 49: 987-995.

14. Mohiuddin $M$, Regine WF, J ohn WJ, et al. Preoperative chemoradiation in fixed distal rectal cancer: dose time factors for pathological complete response. Int J Radiat Oncol Biol Phys 2000; 46: 883-888.

15. Heald RJ, Moran BJ, Ryall RDH, Sexton R, MacFarlane JK. Rectal cancer: the Basingstoke experiene of total mesorectal excision, 1978-1997. Arch Surg 1998; 133: 894-899.

16. Kapiteijn E, Marijnen CAM, Nagtegaal ID, et al. Preoperative radiotherapy combined with total mesorectal excision for resectable rectal cancer. N Engl J Med 2001; 345: 638-646.

17. Russell AH, Harris J, Rosenberg PJ, et al. Anal sphincter conservation for patients with adenocarcinoma of the distal rectum: Long-term results of radiation therapy oncology group protocol 89-02. Int J Radiat Oncol Biol Phys 2000; 46: 313-322.

18. Martín de Vidales C, Cerezo L, Vázquez de la Torre M, et al. Estudio comparativo en el adenocarcinoma de recto: Radioterapia preoperatoria o postoperatoria. Rev Esp Enf Digest 1995; 87: 199-204.

19. Mohiuddin M, Ahmed MM. Critical issues in the evolving management of rectal cancer. Semin Oncol 1997; 24: $732-744$.

20. Mohiuddin M, Marks G, Bannon J. High-dose preoperative radiation and full thickness local excision: A new option for selected T3 distal rectal cancers. Int J Radiat Oncol Biol Phys 1994; 30: 845-849.

21. Minsky BD, Cohen AM, Enker WE, et al. Preoperative 5-FU, low-dose leucovorin and radiation therapy for locally advanced and unresectable rectal cancer. Int J Radiat Oncol Biol Phys 1997; 37: 289-295.

22. Marsh RW, Chu NM, Mendenhall WM, et al. Preoperative treatment of patients with locally advanced unresectable rectal adenocarcinoma utilizing continuous chronobiologically shaped 5-fluorouracil infusion and radiation therapy. Cancer 1996; 78: 217-225.

23. De la Torre A, Ramos S, Valcárcel F, et al. Phase II study of radiochemotherapy with UFT and low-dose oral leucovorin in patients with unresectable rectal cancer. Int J Radiat Oncol Biol Phys 1999; 45: 629-634.

24. François Y, Nemoz CJ, Baulieux J, et al. Influence of the interval between preoperative radiation therapy and surgery on downstaging and on the rate of sphinctersparing surgery for rectal cancer: The Lyon R90-01 randomized trial. J Clin Oncol 1999; 17: 2.396-2.042. 